\title{
FUNCTIONS SATISFYING A WEIGHTED AVERAGE PROPERTY. II ( $\left.{ }^{1}\right)$
}

\author{
ANIL KUMAR BOSE
}

Introduction. In the previous paper [1] we were interested in characterizing the class of functions, defined in a given region (open connected set) $R$ of the $n$-dimensional euclidean space $E_{n}$, which satisfy the following Weighted Average Property (W.A.P.):

$$
\begin{aligned}
& u(P)=\frac{\int_{B(P, r)} u w d \rho}{\int_{B(P, r)} w d \rho}, \quad P \in R, \\
& u(P)=\frac{\int_{S(P, r)} u w d \sigma}{\int_{S(P, r)} w d \sigma},
\end{aligned}
$$

where $B(P, r)$ and $S(P, r)$ denote any ball and its surface with the point

$$
P=P\left(x_{1}, x_{2}, \cdots, x_{n}\right),
$$

for its center and radius $r$ which lies in $R ; d \rho$ and $d \sigma$ stand for the usual Lebesgue measure of $B$ and $S$ and $w$ is a weight function (W.F.) defined in $R$.

For convenience we state here again the definitions of weight functions and functions satisfying a W.A.P.

Definition. $w$ is a W.F. in $R$ means that

(a) $w$ is a nonnegative, real-valued function defined in $R$, and

(b) $w$ is locally summable in $R$, i.e., if $P \in R$ and $0<r<d(P, T), T$ being the boundary of $R$, then the Lebesgue integral $\int_{B(P, r)} w d \rho$ over $B(P, r)$ exists and $\int_{B(P, r)} w d \rho>0$.

Definition. A real-valued function $u$ is said to satisfy the W.A.P. with respect to a W.F., $w$ in $R$ provided $u w$ is locally summable in $R$ and $u$ satisfies the property (0.1) for each ball $B(P, r)$ whose closure lies in $R$.

\section{DEFINITION.}

$$
R^{*}=\{(P, r): P \in R \text { and } 0<r<d(P, T)\} .
$$

Received by the editors November 6, 1965.

(1) This research was supported, in part, by the U. S. Army Research Office, Durham, N. C. 
Suppose now that $R$ be a region in $E_{n}$ and $w$ be a W.F. defined in $R$. Let $S(w, R)$ denote the class of all functions satisfying W.A.P. with respect to $w$ in $R$.

In the previous paper [1] we proved the following theorems and corollaries:

THEOREM 2*.

(i) If the W.F. $w$ belongs to class $C^{m}(R)$, where $m$ is a nonnegative integer and $u \in S(w, R)$, then $u \in C^{m+1}(R)$.

(ii) If $w$ be infinitely differentiable in $R$ and $u \in S(w, R)$, then $u$ is infinitely differentiable in $R$.

(iii) If $w$ be analytic in $R$ and $u \in S(w, R)$, then $u$ is analytic in $R$.

REMARK $1 *$.

(0.3) Constant functions belong to $S(w, R)$.

$(0.4) S(w, R)$ is a linear space over the reals and $\operatorname{dim} S(w, R) \geqq 1$.

(0.5) If $w$ be a nonzero constant, then $S(w, R)$ is the class of all harmonic functions defined on $R$.

REMARK $2^{*}$. In proving Theorem $2^{*}$ the following was shown:

If $w \in C^{1}(R)$ and $u \in S(w, R)$, then

$$
u_{x_{i}}(P)=\frac{\int_{B(P, r)} u_{x_{i}} w d \rho}{\int_{B(P, r)} w d \rho}+\frac{\int_{B(P, r)} u w_{x_{i}} d \rho-u(P) \int_{B(P, r)} w_{x_{i}} d \rho}{\int_{B(P, r)} w d \rho},
$$

$i=1,2, \cdots, n$, for all $(P, r) \in R^{*}$.

THEOREM 4*. If the W.F. $w \in C^{1}(R)$ and $u \in S(w, R)$, then $u$ satisfies the second order elliptic differential equation

$$
w \Delta u+2 \sum_{i=1}^{n} w_{x_{i}} u_{x_{i}}=0
$$

in $R$, where $\Delta u$ is the Laplacian of $u$.

REMARK 3*. In proving Theorem 4* incidentally the following was shown:

If the W.F. $w \in C^{1}(R)$ and $u \in S(w, R)$, then

$$
\Delta u(P) \cdot \int_{B(P, r)} w d \rho+2 \sum_{i=1}^{n} u_{x_{i}}(P) \cdot \int_{B(P, r)} w_{x_{i}} d \rho=0
$$

for each $(P, r) \in R^{*}$.

THEOREM 5*. Let $w$ be a W.F. defined in $R$ and $\lambda$ be a real number such that $w \in C^{2}(R)$ and is a solution of the differential equation

$$
\Delta F+\lambda F=0
$$

in $R$. Then a necessary and sufficient condition that $u \in S(w, R)$ is that 
(i) $u \in C^{2}(R)$ and

(ii) $u$ satisfies the differential equation

$$
w \Delta u+2 \sum_{i=1}^{n} u_{x_{i}} w_{x_{i}}=0
$$

in $R$.

REMARK 4*. In proving Theorem $5^{*}$ incidentally the following was shown: If the W.F. $w \in C^{2}(R)$ and is a solution of $(0.9)$, then $w(P)>0$ for all $P$ in $R$.

Corollary 1*. Let $w$ be a W.F. defined in $R$ and $\lambda$ be a real number such that $w \in C^{2}(R)$ and is a solution of the differential equation

$$
\Delta F+\lambda F=0
$$

in $R$. Then the following are true:

(a) If $f \in C^{2}(R)$ and is a solution of $(0.11)$, then $f / w \in S(w, R)$.

(b) If $u \in S(w, R)$, then $u w \in C^{2}(R)$ and is a solution of (0.11).

Corollary 2*. Let $w$ be a W.F. defined in a region $R$ of $E_{n}(n>1)$ and $\lambda a$ real number such that $w \in C^{2}(R)$, and is a solution of the equation

$$
\Delta F+\lambda F=0
$$

in $R$. Then $S(w, R)$ is infinite dimensional.

REMARK 5*. In $E_{1}$ the following can be proved easily:

If the W.F. $w \in C^{1}(R)$, then

(i) $1 \leqq \operatorname{dim} S(w, R) \leqq 2$

(ii) $\operatorname{dim} S(w, R)=2$ if and only if $w \in C^{2}(R)$ and is a solution of

$$
\frac{d^{2} w}{d x^{2}}+\lambda \frac{d w}{d x}=0
$$

in $R$, for some real constant $\lambda$.

The importance of the weight functions which are solutions of the equation

$$
\Delta w+\lambda w=0
$$

has been demonstrated in the previous paper [1].

In this paper we propose to deduce in terms of the W.A.P. a necessary and sufficient condition for a W.F. to be a solution of (0.13). From this result we will prove our main theorem of $\S \mathrm{I}$, namely, " $S(w, R)$ is infinite dimensional if and only if $w$ is a solution of $(0.13) . "\left({ }^{2}\right)$

In $§$ II we will consider derivatives of functions belonging to $S(w, R)$ and deduce necessary and sufficient conditions for these derivatives to belong to $S(w, R)$.

(2) Added in proof. True only in $E_{2}$. See also footnote (3). 
Here again we will emphasize the role of weight functions which are solutions of (0.13), specially the exponential weight functions $\exp \left\{\sum_{i=1}^{n} a_{i} x_{i}\right\}$.

I. The differential equation $\Delta w+\lambda w=0$.

THEOREM 1. Let $w$ be a W.F. belonging to class $C^{1}(R)$. A necessary and sufficient condition that $w \in C^{2}(R)$ and is a solution of

$$
\Delta w+\lambda w=0
$$

in $R$, where $\lambda$ is some real constant, is that

(i) $w(P)>0$ for all $P\left(x_{1}, x_{2}, \cdots, x_{n}\right)$ in $R$ and

(ii) $w_{x_{i}} / w \in S(w, R)$ for $i=1,2, \cdots, n$.

Proof. Necessity. Let $w \in C^{2}(R)$ and be a solution of (1.0). Then $w$ is analytic in $R$ and each of the partial derivatives $w_{x_{i}}, i=1,2, \cdots, n$, is also a solution of (1.0). Again by Remark $4^{*}, w(P)>0$ for all $P=P\left(x_{1}, x_{2}, \cdots, x_{n}\right)$ in $R$. Hence by Corollary $1^{*}, w_{x_{i}} / w \in S(w, R)$ for $i=1,2, \cdots, n$.

Sufficiency. Suppose that $w(P)>0$ for all $P=P\left(x_{1}, x_{2}, \cdots, x_{n}\right)$ in $R$ and $w_{x_{i}} / w \in S(w, R)$ for $i=1,2, \cdots, n . w_{x_{i}} / w=F^{i} \in S(w, R)$ implies by Theorem $2^{*}$ that $F^{i} \in C^{2}(R)$ and $w \in C^{3}(R)$. Also by Theorem $4^{*}, F^{i}$ is a solution of the differential equation

$$
w \Delta F^{i}+2 \sum_{j=1}^{n} F_{x}^{i} w_{x_{j}}=0,
$$

$i=1,2, \cdots, n$. Again $F^{i} w=w_{x_{i}}$ implies that

$$
\Delta w+\lambda w=0
$$

where

$$
\lambda=-\sum_{i=1}^{n}\left\{F_{x_{i}}^{i}+\left(F^{i}\right)^{2}\right\}
$$

Clearly $\lambda$ belongs to class $C^{1}(R)$ and

$$
\frac{\partial \lambda}{\partial x_{j}}=\lambda_{x_{j}}=-\sum_{i=1}^{n}\left\{F_{x_{i} x_{j}}^{i}+2 F^{i} F_{x_{j}}^{i}\right\}, \quad j=1,2, \cdots, n .
$$

Using

$$
F_{x_{j}}^{i}=F_{x_{i}}^{j}, F_{x_{j} x_{i}}^{i}=F_{x_{i} x_{i}}^{j}, \quad i, j=1,2, \cdots, n
$$

we have

$$
w \lambda_{x_{j}}=-\sum_{i=1}^{n}\left\{w F_{x_{i} x_{i}}^{j}+2 F_{x_{i}}^{j} w_{x_{i}}\right\}=-\left\{w \Delta F^{j}+2 \sum_{i=1}^{n} F_{x_{i}}^{j} W_{x_{i}}\right\}=0
$$

Since

$$
w \neq 0 \text { in } R, \lambda_{x_{j}}=0 \text { in } R \text { for } j=1,2, \cdots, n .
$$


Therefore $\lambda$ is a constant. This completes the proof.

Dimension of $S(w, R)$.

THEOREM 2. Let $R$ be a region in $E_{2}$ and $w$ be a positive W.F. belonging to class $C^{1}(R)$. Then $S(w, R)$ is infinite dimensional if and only if $w$ belongs to class $C^{2}(R)$ and is a solution of

$$
\Delta w+\lambda w=0
$$

in $R$ for some real constant $\lambda$. Furthermore, if $S(w, R)$ is finite dimensional then $1 \leqq \operatorname{dim} S(w, R) \leqq 2$.

Proof. If $w \in C^{2}(R)$ and is a solution of $(1.3)$ then $S(w, R)$ is infinite dimensional by Corollary $2^{*}$. So now suppose that $w$ be a positive W.F. belonging to class $C^{1}(R)$ such that $\operatorname{dim} S(w, R)>2$. Then there exists two nonconstant functions $u_{1}$ and $u_{2}$ both belonging to $S(w, R)$ such that $1, u_{1}, u_{2}$, are linearly independent over $R$. By Theorem 4* and Remark 3* we have

$$
w \Delta u_{i}+2\left(w_{x} u_{i x}+w_{y} u_{i y}\right)=0, i=1,2, .
$$

in $R$ and

$$
\Delta u_{i}(P)+2 u_{i x}(P)\left[\frac{\int_{B(P, r)} w_{x} d \rho}{\int_{B(P, r)} w d \rho}\right]+2 u_{i y}(P)\left[\frac{\int_{B(P, r)} w_{y} d \rho}{\int_{B(P, r)} w d \rho}\right]=0
$$

for each $(P, r) \in R^{*}$. Therefore we have

$$
u_{i x}(P)\left[\frac{w_{x}(P)}{w(P)}-\frac{\int_{B(P, r)} w_{x} d \rho}{\int_{B(P, r)} w d \rho}\right]+u_{i y}(P)\left[\frac{w_{y}(P)}{w(P)}-\frac{\int_{B(P, r)} w_{y} d \rho}{\int_{B(P, r)} w d \rho}\right]=0
$$

for each $(P, r) \in R^{*}, i=1,2$.

Let $R_{1}$ be the subset of $R$ to which a point $P$ belongs if and only if the Jacobian

$$
J_{12}=\frac{D\left(u_{1}, u_{2}\right)}{D(x, y)}=\left|\begin{array}{ll}
u_{1 x} & u_{1 y} \\
u_{2 x} & u_{2 y}
\end{array}\right|
$$

does not vanish at $P . R_{1}$ is a dense subset of $R$. For, if not, then there exists a point $P$ in $R-R_{1}$ and a neighborhood $N(P)$ of the point $P$ lying in $R$ such that the Jacobian $J_{12}$ vanishes identically in $N(P)$. If at least one of the minors, say $u_{1 x}$, is not identically zero in $N(P)$, then there is a point $Q$ and a neighborhood $N(Q)$ of the point $Q$ lying in $N(P)$ such that $u_{1 x} \neq 0$ in $N(Q)$. Hence there exists a functional relation $u_{2}=\phi\left(u_{1}\right)$ valid in $N(Q)$. Since each of $u_{1}$ and $u_{2}$ belong 
to $C^{2}(R)$ and $u_{1 x} \neq 0$ in $N(Q), \phi$ is twice continuously differentiable and we have

or

$$
w \Delta u_{2}+2 u_{2 x} w_{x}+2 u_{2 y} w_{y}=w \cdot \frac{d^{2} \phi}{d u_{1}^{2}}\left\{\left(u_{1 x}\right)^{2}+\left(u_{1 y}\right)^{2}\right\}=0
$$

$$
\frac{d^{2} \phi}{d u_{1}^{2}}=0
$$

Hence $u_{2}=c_{1} u_{1}+c_{2}$, in $N(Q)$, where $c_{1}$ and $c_{2}$ are real constants. This means by the maximum principle of the elliptic equation

$$
w \Delta u+2 w_{x} u_{x}+2 w_{y} u_{y}=0
$$

that $u_{2}=c_{1} u_{1}+c_{2}$ in $R$, contradicting the linear independence assumption. Therefore every minor of $J_{12}$ is identically zero in $N(P)$ implying that each of $u_{1}$ and $u_{2}$ is constant in $N(P)$ and hence in $R$ contradicting again our hypothesis. Therefore $R_{1}$ is a dense subset of $R$. Now to prove our theorem consider the equations (1.6). Clearly

$$
\begin{aligned}
& \int_{B(P, r)} w_{x} d \rho / \int_{B(P, r)} w d \rho=w_{x}(P) / w(P) \\
& \int_{B(P, r)} w_{y} d \rho / \int_{B(P, r)} w d \rho=w_{y}(P) / w(P)
\end{aligned}
$$

for each point $P$ in $R_{1}$ and each $r$ satisfying $0<r<d(P, T)$. Since $R_{1}$ is dense, we conclude that the relations (1.8) and (1.9) are true for each $(P, r) \in R^{*}$. This means that each of the functions $w_{x} / w$ and $w_{y} / w$ belongs to $S(w, R)$. Hence by Theorem 1 there exists a real constant $\lambda$ such that $w \in C^{2}(R)$ and is a solution of (1.3). Therefore $S(w, R)$ is infinite dimensional by Corollary $2^{*}$.

It is clear also that if $S(w, R)$ is finite dimensional then $1 \leqq \operatorname{dim} S(w, R) \leqq 2$.

THEOREM $3\left({ }^{3}\right)$. Let $R$ be a region in $E_{n}(n>2)$ and $w$ be a positive W.F. belonging to class $C^{1}(R)$. Then $S(w, R)$ is infinite dimensional if and only if $w$ belongs to class $C^{2}(R)$ and is a solution of

$$
\Delta w+\lambda w=0
$$

in $R$, for some real constant $\lambda$. If $S(w, R)$ is finite dimensional then

$$
1 \leqq \operatorname{dim} S(w, R) \leqq 2 n-1 .
$$

Proof. For simplicity we will give the proof for $n=4$. Proof for the general case is quite similar.

(3) Added in proof. Theorem 3 is not possibly true. Mr. David Stanford of Denison University, Granville, Ohio, seems to have a counterexample. The author hopes to clarify this point in a future paper. 
If $w \in C^{2}(R)$ and is a solution of $\Delta w+\lambda w=0$, for some real constant $\lambda$, then by Corollary $2^{*}, S(w, R)$ is infinite dimensional. So now suppose that $w$ be a positive W.F. belonging to class $C^{1}(R)$ such that $\operatorname{dim} S(w, R)>2 \cdot 4-1=7$. Then there exists nonconstant functions $u_{1}, u_{2}, \cdots, u_{7}$ all belonging to $S(w, R)$ such that $1, u_{1}, u_{2}, \cdots, u_{7}$ are linearly independent over $R$. As in Theorem 2, we have by Theorem $4^{*}$ and Remark $3^{*}$ the system of 7 equations:

$$
\sum_{i=1}^{n} u_{j x_{i}}(P)\left[w_{x_{i}}(P) / w(P)-\int_{B(P, r)} w_{x_{i}} d \rho / \int_{B(P, r)} w d \rho\right]=0,
$$

$j=1,2, \cdots, 7$; for each $(P, r) \in R^{*}$.

Let $\left(j_{1}, j_{2}, j_{3}, j_{4}\right)$ be a combination of four distinct integers taken from the seven positive integers $1,2, \cdots, 7$. The system of equations (1.10) gives rise to ${ }_{7} C_{4}=35$ Jacobians of the form

$$
J_{j_{1} j_{2} j_{3} j_{4}}=\frac{D\left(u_{j_{1}}, u_{j_{2}}, u_{j_{3}}, u_{j_{4}}\right)}{D\left(x_{1}, x_{2}, x_{3}, x_{4}\right)} .
$$

Let $R_{1}$ be the subset of $R$ to which a point $P$ belongs if and only if at least one of the ${ }_{7} C_{4}=35 \mathrm{Jacobians}\left\{J_{j_{1} j_{2} j_{3} j_{4}}\right\}$ does not vanish at $P . R_{1}$ is a dense subset of $R$. For, if not, then there exists a point $P$ and a neighborhood $N(P)$ of the point $P$ lying in $R$ such that each of the 35 Jacobians $\left\{J_{j_{1} j_{2} j_{3} j_{4}}\right\}$ vanishes identically in $N(P)$. This again means that every first, second, and third minor of each of the 35 Jacobians $\left\{J_{j_{1} j_{2} j_{3} j_{4}}\right\}$ vanishes identically in $N(P)$. For, if possible, let one of the first minors of $J_{1234}$, say $D\left(u_{1}, u_{2}, u_{3}\right) / D\left(x_{1}, x_{2}, x_{3}\right)$, does not vanish identically in $N(P)$. Then there is a point $Q$ and a neighborhood $N(Q)$ of the point $Q$ lying in $N(P)$ such that $D\left(u_{1}, u_{2}, u_{3}\right) / D\left(x_{1}, x_{2}, x_{3}\right) \neq 0$ in $N(Q)$. Hence considering also the Jacobians $J_{1235}, J_{1236}, J_{1237}$, there exists functional relations

$$
\begin{aligned}
& u_{4}=\phi_{4}\left(u_{1}, u_{2}, u_{3}\right), u_{5}=\phi_{5}\left(u_{1}, u_{2}, u_{3}\right), \\
& u_{6}=\phi_{6}\left(u_{1}, u_{2}, u_{3}\right), u_{7}=\phi_{7}\left(u_{1}, u_{2}, u_{3}\right)
\end{aligned}
$$

each valid in $N(Q)$. Also there is a functional relation

$$
\phi\left(u_{4}, u_{5}, u_{6}, u_{7}\right)=0,
$$

valid in $N(P)$.

Therefore (1.11) and (1.12) imply that there is a functional relation

$$
\psi\left(u_{1}, u_{2}, u_{3}\right)=0,
$$

valid in $N(Q)$ contradicting the fact that $D\left(u_{1}, u_{2}, u_{3}\right) / D\left(x_{1}, x_{2}, x_{3}\right) \neq 0$ in $N(Q)$. Hence every first minor of each of the 35 Jacobians vanishes identically in $N(P)$. If possible, suppose now that one of the second minors of $J_{1234}$, say, $D\left(u_{1}, u_{2}\right) / D\left(x_{1}, x_{2}\right)$, is not identically zero in $N(P)$. Then there exists a point $Q$ and a neighborhood $N(Q)$ of the point $Q$ lying in $N(P)$ such that $D\left(u_{1}, u_{2}\right) / D\left(x_{1}, x_{2}\right)$ 
$\neq 0$ in $N(Q)$. Hence, considering also the Jacobian $J_{1256}$, there exists functional relations

$$
u_{3}=\phi_{3}\left(u_{1}, u_{2}\right), u_{4}=\phi_{4}\left(u_{1}, u_{2}\right), u_{5}=\phi_{5}\left(u_{1}, u_{2}\right), u_{6}=\phi_{6}\left(u_{1}, u_{2}\right)
$$

valid in $N(Q)$. (1.12) and (1.13) lead to functional relation $\psi\left(u_{1}, u_{2}\right)=0$ valid in $N(Q)$, contradicting the nonvanishing of $D\left(u_{1}, u_{2}\right) / D\left(x_{1}, x_{2}\right)$ in $N(Q)$. Hence every second minor of each of the 35 Jacobians $\left\{J_{j_{1} j_{2} j_{3} j_{4}}\right\}$ vanishes identically in $N(P)$. Finally suppose that one of the third minors of $J_{1234}$, say $\partial u_{1} / \partial x_{1}$ is not identically zero in $N(P)$. Then there exists functional relations of the form

$$
u_{2}=\phi_{2}\left(u_{1}\right), \quad u_{3}=\phi_{3}\left(u_{1}\right), \quad u_{4}=\phi_{4}\left(u_{1}\right),
$$

valid in some neighborhood $N(Q)$ of a point $Q$ lying in $N(P)$. Now applying similar arguments as in Theorem 2 , we get a relationship $u_{2}=c_{1} u_{1}+c_{2}$ valid in $R$, where $c_{1}$ and $c_{2}$ are real constants which contradicts the linear independence assumption. Therefore every third minor of each of the 35 Jacobians $\left\{J_{j_{1} j_{2} j_{3} j_{4}}\right\}$ vanishes identically in $N(P)$, which means that each of the functions $u_{1}, u_{2}, u \cdots, u_{7}$ is constant in $N(Q)$ and hence, by the maximum principle of the elliptic equation (0.10), in $R$, implying again a contradiction. Therefore $R_{1}$ is a dense subset of $R$.

Now arguing similarly as in Theorem 2 , we conclude that each of the functions $w_{x_{i}} / w, i=1,2,3,4$, belong to $S(w, R)$. Hence by Theorem 1 , there exists a real constant $\lambda$ such that $w \in C^{2}(R)$. and is a solution of $(1.0)$ in $R$. Hence $S(w, R)$ is infinite dimensional by Corollary $2^{*}$. It is also clear that if $S(w, R)$ is finite dimensional then $1 \leqq \operatorname{dim} S(w, R) \leqq 2 \cdot 4-1=7$.

II. Derivatives of functions satisfying W.A.P. It is known that derivatives of harmonic functions, defined in a given region $R$ of $E_{n}$, are also harmonic in that region. But this is not, in general, true for functions satisfying W.A.P. unless they satisfy a similar W.A.P. with respect to the derivatives of the W.F. For example, consider the W.F., $w(x, y)=x+y$, defined in $R$, where $R$ is the first quadrant of the plane $E_{2}$. The function $u(x, y)=x^{2}-4 x y+y^{2}$ is a solution of

$$
w \Delta u+2\left(w_{x} u_{x}+w_{y} u_{y}\right)=0
$$

in $R$. Also $u \in C^{2}(R)$. Since $w \in C^{2}(R)$ and is a solution of $\Delta w=0$ in $R$, by Theorem $5^{*}, u \in S(w, R)$. But $u_{x}=2 x-4 y$ and $u_{y}=-4 x+2 y$, do not satisfy (2.0) and hence cannot satisfy W.A.P. with respect to $w$ in $R$.

On the other hand $w(x, y)=\exp (x+y)$ is a W.F. in $E_{2} . w \in C^{2}\left(E_{2}\right)$ and is a solution of

$$
\Delta w-2 w=0
$$

in $E_{2}$. The function $u(x, y)=x^{2}-2 x y+y^{2}-x-y$ belongs to class $C^{2}\left(E_{2}\right)$ and is a solution of 


$$
w \Delta u+2 w_{x} u_{x}+2 w_{y} u_{y}=0
$$

in $E_{2}$. Hence by Theorem $5^{*}, u \in S\left(w, E_{2}\right)$. Also each of the derivatives $u_{x}=2 x-2 y-1, u_{y}=-2 x+2 y-1$ belongs to class $C^{2}\left(E_{2}\right)$ and is a solution of (2.0). Therefore each of the derivatives $u_{x}$ and $u_{y}$ belongs to $S\left(w, E_{2}\right)$.

THEOREM 4. Let $R$ be a region in $E_{n}$ and $w$ be a W.F. belonging to class $C^{1}(R)$. If $u \in S(w, R)$, then a necessary and sufficient condition that the partial derivative $\partial u / \partial x_{i}=u_{x_{i}}, 1 \leqq i \leqq n$, will belong to $S(w, R)$ is that

$$
\int_{B(P, r)} u w_{x_{i}} d \rho=u(P) \int_{B(P, r)} w_{x_{i}} d \rho
$$

for each $(P, r) \in R^{*}$.

Proof. By Remark 2*, the partial derivative $u_{x_{i}}$ satisfies the relation

$$
u_{x_{i}}(P) \int_{B(P, r)} w d \rho=\int_{B(P, r)} u_{x_{i}} w d \rho+\left[\int_{B(P, r)} u w_{x_{i}} d \rho-u(P) \int_{B(P, r)} w_{x_{i}} d \rho\right]
$$

for each $(P, r) \in R^{*}$. It is clear from (2.2) that the theorem is true.

REMARK 1. If $w$ be a positive constant, then $S(w, R)$ is the class of all harmonic functions defined in $R$ and $w_{x_{i}}=0$, for $i=1,2, \cdots, n$, implies that the relation (2.1) is true for constant W.F., which simply means that derivatives of harmonic functions are also harmonic as is well known.

THEOREM 5. Let the W.F. $w$ belong to class $C^{2}(R)$ and be solution of

$$
\Delta w+\lambda w=0
$$

in $R$, where $\lambda$ is a real constant. If $u \in S(w, R)$ then these are equivalent:

(i) $\quad u_{x_{i}}, 1 \leqq i \leqq n$, belongs to $S(w, R)$

(ii) $u w_{x_{i}} / w$ belongs to $S(w, R)$

(iii) $u w_{x_{i}}$ is a solution of (2.3) in $R$

(iv) $u_{x_{i}} w$ is a solution of (2.3) in $R$

(v) $u$ is a solution of

$$
w_{x_{i}} \Delta u+2 \sum_{j=1}^{n} u_{x_{j}} w_{x_{i} x_{j}}=0
$$

in $R$.

Proof. By hypothesis and from Theorem 2*, each of $u$ and $w$ is analytic in $R$. Also, by Theorem $1, w(P)>0$ for each point $P=P\left(x_{1}, x_{2}, \cdots, x_{n}\right)$ in $R$ and each of the functions $w_{x_{i}} / w, i=1,2, \cdots, n$, belongs to $S(w, R)$.

Now suppose that (i) is true. Using Remark $3^{*}$ we can write

or

$$
u_{x_{i}}(P) \int_{B(P, r)} w d \rho=\int_{B(P, r)}\left\{u_{x_{i}}+\left(u w_{x_{i}}\right) / w\right\} w d \rho-u(P) \int_{B(P, r)}\left\{w_{x_{i}} / w\right\} w d \rho,
$$




$$
\left\{u_{x_{i}}(P)+u(P) w_{x_{i}}(P) / w(P)\right\} \int_{B(P, r)} w d \rho=\int_{B(P, r)}\left\{u_{x_{i}}+\left(u w_{x_{i}}\right) / w\right\} w d \rho
$$

for each $(P, r) \in R^{*}$ which implies that $u_{x_{i}}+u w_{x_{i}} / w \in S(w, R)$. Since $S(w, R)$ is a linear space, $u w_{x_{i}} / w \in S(w, R)$. Hence (i) implies (ii). Next suppose that (ii) is true. Then by Corollary $1^{*}, u w_{x_{i}}$ is a solution of (2.3). Therefore (ii) implies (iii). Now suppose that (iii) is true. By hypothesis and from Corollary $1^{*}$, $u w$ is a solution of (2.3) and hence $(u w)_{x_{i}}=u_{x_{i}} w+u w_{x_{i}}$ is also a solution of (2.3). We conclude from the linearity of (2.3) that $u_{x_{i}} w$ is a solution of (2.3). Thus (iii) implies (iv).

Next suppose that (iv) is true. We have

$$
\Delta\left(u_{x_{i}} w\right)+\lambda\left(u_{x_{i}} w\right)=0 .
$$

Also by hypothesis $\Delta w+\lambda w=0$ and

$$
w \Delta u+2 \sum_{j=1}^{n} u_{x_{j}} w_{x_{j}}=0 .
$$

Differentiation of (2.6) with respect to $x_{i}$ yields

$$
\left\{w_{x_{i}} \Delta u+2 \sum_{j=1}^{n} u_{x_{j}} w_{x_{i} x_{j}}\right\}+\left\{\Delta\left(u_{x_{i}} w\right)+\lambda\left(u_{x_{i}} w\right)\right\}=0
$$

or

$$
w_{x_{i}} \Delta u+2 \sum_{j=1}^{n} u_{x_{i}} w_{x_{i} x_{i}}=0 .
$$

Therefore (iv) implies (v).

Finally suppose that $(v)$ is true. Now differentiation of (2.6) with respect to $x_{i}$ gives

$$
\left\{w_{x_{i}} \Delta u+2 \sum_{j=1}^{n} u_{x_{j}} w_{x_{i} x_{j}}\right\}+\left\{w \Delta u_{x_{i}}+2 \sum_{j=1}^{n} u_{x_{i} x_{j}} w_{x_{j}}\right\}=0 \text {. }
$$

Therefore we have $w \Delta u_{x_{i}}+2 \sum_{j=1}^{n} u_{x_{i} x_{j}} w_{x_{j}}=0$, which by Theorem $5 *$ means that $u_{i} \in S(w, R)$. This completes the cycle.

Exponential Weight Functions, Definition. Weight functions of the form

$$
w\left(x_{1}, x_{2}, \cdots, x_{n}\right)=k \exp \left(a_{1} x_{1}+a_{2} x_{2}+\cdots+a_{n} x_{n}\right),
$$

$\left(x_{1}, x_{2}, \cdots, x_{n}\right) \in E_{n}$, where $a_{i}$ 's are real constants and $k$ is a positive real constant, are called exponential weight functions.

Properties of exponential weight functions. If $w$ be an exponential W.F., then

(2.7) $w$ is an analytic W.F. in any subregion $R$ of $E_{n}$,

(2.8) $w$ is a solution of $\Delta w+\lambda w=0$ in $E_{n}$, where $\lambda=-\sum_{i=1}^{n} a_{i}^{2}$.

(2.9) $w_{x_{i}} / w=a_{i}, i=1,2, \cdots, n$. 
The differential equation $\Delta u+2 \sum_{i=1}^{n} a_{i} u_{x_{i}}=0$.

THEOREM 6. For an exponential W.F. $w\left(x_{1}, x_{2}, \cdots, x_{n}\right)=k \exp \left(\sum_{i=1}^{n} a_{i} x_{i}\right), a$ necessary and sufficient condition that $u \in S(w, R)$ is that $u \in C^{2}(R)$ and is a solution of

$$
\Delta u+2 \sum_{i=1}^{n} a_{i} u_{x_{i}}=0
$$

in $R$.

Proof. The theorem follows at once from Theorem 5*.

THEOREM 7. Let $w\left(x_{1}, x_{2}, \cdots, x_{n}\right)=k \exp \left(\sum_{i=1}^{n} a_{i} x_{i}\right)$ be an exponential W.F. If $u \in S(w, R)$, then

(i) $u$ is analytic in $R$ and

(ii) if $m$ be a positive integer and $D^{m} u$ be any mth order partial derivative of $u$, then $D^{m} u \in S(w, R)$.

Proof. Part (i) is an immediate consequence of Theorem $2^{*}$. Again $S(w, R)$ is a linear space and $u \in S(w, R)$ implies that each of the functions $u w_{x_{i}} / w=a_{i} u$, $i=1,2, \cdots, n$, also belongs to $S(w, R)$. Hence by Theorem 5 each of the derivatives $u_{x_{i}}, i=1,2, \cdots, n$, belongs to $S(w, R)$. Applying mathematical induction, we see that the theorem is true.

Characterisation of exponential W.F. Property (2.9) and part (ii) of Theorem 7 characterises exponential weight function in the sense of the following theorem:

THEOREM 8. Let $w$ be a W.F. belonging to class $C^{2}(R)$ and is a solution of $\Delta w+\lambda w=0$ for some real constant. Then the following are equivalent:

(i) If $u \in S(w, R)$, then each of the derivatives $u_{x_{i}}, i=1,2, \cdots, n$, also belongs to $S(w, R)$.

(ii) $w$ is an exponential W.F.

Proof. By Theorem 1, $w(P)=w\left(x_{1}, x_{2}, \cdots, x_{n}\right)>0$ for all $P\left(x_{1}, x_{2}, \cdots, x_{n}\right)$ in $R$ and each of the functions $w_{x_{i}} / w \in S(w, R), i=1,2, \cdots, n$. Suppose that (i) is true. Then each of the partial derivatives $\left(\partial / \partial x_{i}\right)\left(w_{x_{i}} / w\right), i=1,2, \cdots, n$, also belongs to $S(w, R)$. Therefore by Theorem 5 each of the functions $\left(w_{x_{i}} / w\right)^{2}$ belongs to $S(w, R)$. Let $P_{0} \in R$. Since $S(w, R)$ is a linear space, each of the functions $F_{i}=\left\{w_{x_{i}} / w-w_{x_{i}}\left(P_{0}\right) / w\left(p_{0}\right)\right\}^{2}, i=1,2, \cdots, n$, also belongs to $S(w, R)$. Since $F_{i}$ has a minimum (zero) at $P_{0}$ in $R$, it follows from the minimum-principle of the differential equation

$$
w \Delta u+2 \sum_{i=1}^{n} w_{x_{i}} u_{x_{i}}=0
$$

that $w_{x_{i}}=a_{i} w, i=1,2, \cdots, n$, where $a_{i}$ 's are real constants. Hence $w\left(x_{1}, x_{2}, \cdots, x_{n}\right)$ 
$=k \exp \left(\sum_{i=1}^{n} a_{i} x_{i}\right)$, for each $\left(x_{1}, x_{2}, \cdots, x_{n}\right) \in R$, where $k$ is a positive real constant. Therefore (i) implies (ii). Now suppose that (ii) is true. Let $w\left(x_{1}, x_{2}, \cdots, x_{n}\right)$ $=k \exp \left(\sum_{i=1}^{n} a_{i} x_{i}\right)$ and $u \in S(w, R)$. Then by Theorem 7 each of the derivatives $u_{x_{i}}, i=1,2, \cdots, n$, belongs to $S(w, R)$. Hence (ii) implies (i). This completes the proof.

\section{BIBLIOGRAPHY}

1. Anil K. Bose, Functions satisfying a weighted average property, Trans. Amer. Math. Soc. 118 (1965), 472-487.

2. Edouard Goursat, $A$ course in mathematical analysis, Vol. I, Dover, New York, 1904.

University of Alabama, Tuscaloosa, Alabama

University OF North Carolina

Chapel Hill, North Carolina 